\title{
Hospitalisation Due to Heart Failure with Gliptins and Universal Label Change (FDA)-Still Justified? A Meta-Analysis of 5 Cardiovascular Outcomes Trials
}

Samit Ghosal ${ }^{1^{*}}$ and Awadhesh Kumar Singh ${ }^{2}$

${ }^{1}$ Consultant Endocrinologist, Nightingale Hospital, Kolkata, India

${ }^{2}$ Consultant Endocrinologist, GD Hospital \& Diabetes Institute, Kolkata, India

“Corresponding author: Dr. Samit Ghosal, MD, MSc, FRCP. Consultant Endocrinologist, Nightingale Hospital, Kolkata, India, Tel: +91-9674328281; E-mail: ramdasghosal@gmail.com

Received date: November 06, 2018; Accepted date: November 22, 2018; Published date: November 29, 2018

Copyright: (c) 2018 Ghosal S, et al. This is an open-access article distributed under the terms of the Creative Commons Attribution License, which permits unrestricted use, distribution, and reproduction in any medium, provided the original author and source are credited.

\begin{abstract}
Introduction: The addition of gliptins in the management of type 2 diabetes was a welcome one due to its neutral impact on weight and lower risk of hypoglycaemia, in addition to moderate anti-hyperglycemic effects. They were also the first anti-hyperglycemic group exposed to the scrutiny of cardiovascular safety assessed through cardiovascular outcomes trial. The disparate signals emanating from the different CVOTs along with recent FDA label change (hospitalisation due to heart failure), prompted us in performing this meta-analysis.
\end{abstract}

Materials and methods: After conducting an extensive database search, we selected 5 CVOTs for this metaanalysis based on a pre-specified set of inclusion criteria. Sufficient caution was exercised in analyzing data heterogeneity and detecting publication bias.

Results: The meta-analysis of the 5 CVOTs with respect to hospitalization due to heart failure resulted in a risk ratio of 1.013 with a $95 \% \mathrm{Cl}$ of $0.857-1.197$. The effect size analysed with the random effect model was statistically non-significant $(P=0.879)$.

Conclusion: This meta-analysis did not find a statistically significant increased risk for hHF in patients with high cardiovascular risk while on a gliptin.

Keywords: Diabetes; Gliptins; Hospitalisation due to heart failure; Cardiovascular outcomes trial; Meta-analysis; FDA

\section{Introduction}

The modern-day medical world is bombarded by a flurry of publications. These evidences (both positive and negative) needs to be incorporated into practice guidelines and recommendations. However, as the popular saying goes "nothing in the world causes so much misery as uncertainty", the recent gliptin label change incorporating hospitalisation due to heart failure (hHF) for the entire class created a similar misery as far as adopting gliptins as an important diabetes management strategy is concerned [1]. The only way forward, is to accumulate more data and clear the uncertainty.

\section{The background story}

It all started with the rosiglitazone story in 2008 [2]. The adverse signal from a cardiovascular point of view prompted regulatory authorities to come up with a recommendation for the industry mandating proof of CVV safety for anti-hyperglycemic therapy [3]. This resulted in a boom of cardiovascular outcomes trial and gliptins were the first to get off the block. The first gliptin trial we came across was SAVOR-TIMI 53 with saxagliptin [4]. Although saxagliptin had a positive cardiovascular $(\mathrm{CV})$ signal when FDA analyzed their pooled phase 2 \& 3 studies, it was limited by very small number of cardiovascular events [5].

Hence a larger CVOT with longer duration of follow up and a prespecified number of cardiovascular events was necessary. The safety of gliptins in diabetic patients with established cardiovascular events or high CV risk was established in this trial. Surprisingly the trial also raised the issue of increased hospitalization due to heart failure (hHF) in the saxagliptin arm compared to the placebo arm. Another CVOT with alogliptin raised similar concern [6]. However, it was the third CVOT (with sitagliptin) which reassured the medical community as far as hHF was concerned [7]. In spite of a statistically non-significant increased hHF rates in EXAMINE trial and a neutral signal from TECOS trial, we saw the regulatory authorities recommending a blanket caution as far as the whole class of agents are concerned including linagliptin [1].

This was an absolutely unexpected finding since there were no previous signals indicative of the same.

\section{A flurry of meta analyses}

A meta-analysis of 9 randomized controlled trials (RCTs) of saxagliptin by Kongwatcharapong et al did suggest an increased heart failure signal $(\mathrm{HR}=1.215,95 \%$ CI 1.028-1.437, $\mathrm{p}=0.022)$ [8]. However, another meta-analysis that was done with RCT's of gliptins including 3 CVOTs, showed inconsistent results on hHF signal (Table 1) [9-12]. 
Page 2 of 4

\begin{tabular}{|l|l|c|c|c|c|}
\hline \multicolumn{1}{|c|}{ Studies analyzed } & \multicolumn{1}{|c|}{$\begin{array}{c}\text { Compared } \\
\text { with }\end{array}$} & $\mathbf{N}$ & HR/OR & $\mathbf{9 5 \%} \mathbf{C l}$ & $\begin{array}{c}\mathbf{P} \\
\text { value }\end{array}$ \\
\hline 3 CVOT only [9] & Placebo & 36,543 & 1.12 & $1.00-1.25$ & 0.05 \\
\hline $\begin{array}{l}5 \quad \mathrm{RCTs} \text { (including 3 } \\
\text { CVOT) [10] }\end{array}$ & Placebo & 37,028 & 1.13 & $1.00-1.26$ & 0.05 \\
\hline $\begin{array}{l}90 \mathrm{RCTs} \text { (including 3 } \\
\text { CVOT) [11] }\end{array}$ & Placebo & 66,730 & 1.11 & $0.99-1.25$ & 0.07 \\
\hline $\begin{array}{l}29 \text { RCTs+3 CVOT } \\
{[12]}\end{array}$ & Placebo/active & 54,640 & 1.13 & $1.01-1.26$ & 0.03 \\
\hline
\end{tabular}

Table 1: Meta-analysis of 3 CVOTs of gliptins with or without RCTs inclusion for hHF.

Since the regulatory bodies are referring to CVOTs as a standardized document to analyze and come up with subsequent recommendations and label changes, we were interested in looking into meta-analysis involving CVOTs only. Prior to the publication of the truncated version of omarigliptin CVOT \& CARMELINA, there were only 3 CVOTs available. A meta-analysis of all these 3 CVOTs revealed a $12 \%$ relative increased risk of hHF with gliptins (95\% CI: 1.00-1.25) [13]. All these data, along with the label change recommendations by FDA led to confusion among clinicians whether gliptins are safe for diabetic patients with established CVD as far as risk of hHF is concerned.

\section{Meta-Analysis of 5 CVOTs}

\section{Materials and Methods}

\section{Search criteria}

We performed a database search for title, abstract \& keywords, without any language restriction. Search terms included "dipeptidyl peptidase-4 inhibitors", "saxagliptin", "alogliptin", "sitagliptin”, "linagliptin", "vildagliptin", "omarigliptin" and "hospitalisation due to heart failure". The search was conducted clubbing the gliptins with hospitalisation due to heart failure. The initial search yielded 507 articles consisting of a combination of citations from Cochrane library, Embase \& PubMed (Figure 1). Further filters included cardiovascular outcomes trial (CVOT), randomized controlled trials (RCTs) and publication dates from 2008 till date. This resulted in 30 trials entering the eligibility phase [14].

The set of articles selected for meta-analysis was subsequently selected based on the inclusion criteria set in advance:

- Patient population studied in each of the trials (n) more than one thousand

- MACE or MACE plus must be the primary end point according to the recommendation to the industry by the regulatory bodies.

- Median duration of follow up more than 12 months.

Ultimately, with the selection criteria mentioned above we selected 5 randomized controlled cardiovascular outcomes trial for analysis [15].

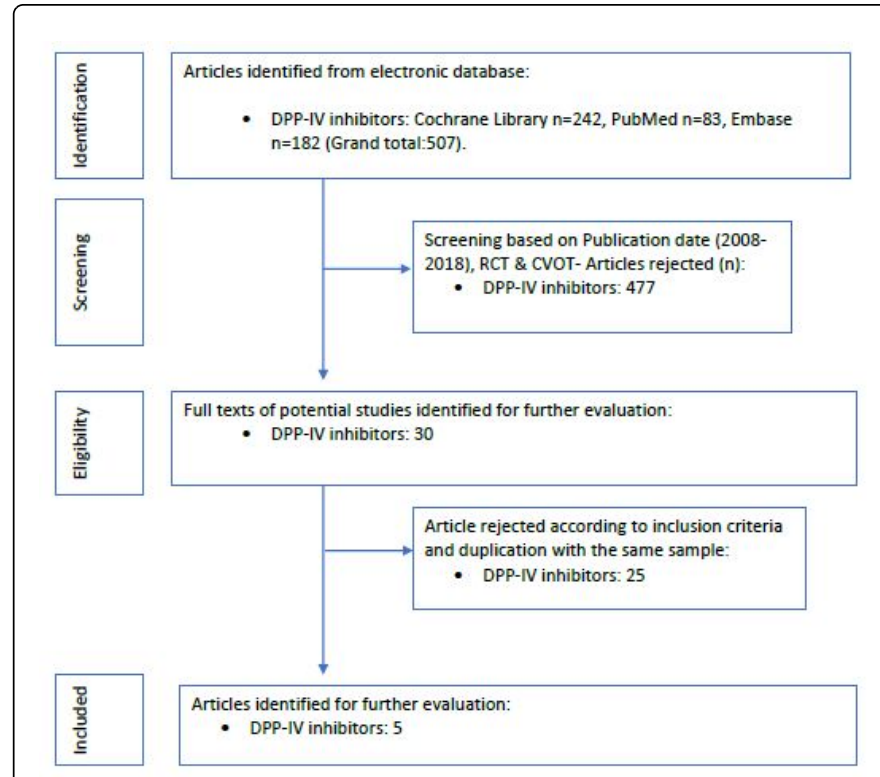

Figure 1: Study Selection Process.

\section{Data Analysis and Results}

The data was analyzed from the 5 selected CVOTs with additional inputs from supplementary materials and analysis of the outcomes data by regulatory authorities. Since, the outcome of interest was hHF, other cardiovascular outcomes were not included in this analysis. We performed the meta-analysis of 5 trials $(n=47,645)$ using comprehensive meta-analysis (CMA) software version 3, Biostat Inc., Englewood, NJ, United States. Since there was a moderate degree of heterogeneity $\left(I^{2}: 61.02\right)$, variable patient population in the trials selected and different weightage to the effect size, a random effect model was used to assess the risk ratio (RR) for the pooled effect size. Heterogeneity was assessed using the Cochrane $\mathrm{Q}$ and Higgin's $\mathrm{I}^{2}$ test and publication bias was assessed by funnel plots.

The pooled analysis of effect size (Risk Ratio) for hHF was not statistically significant (RR:1.013, 95\% CI:0.857-1.197, p value $=0.879$ ) (Figure 2).

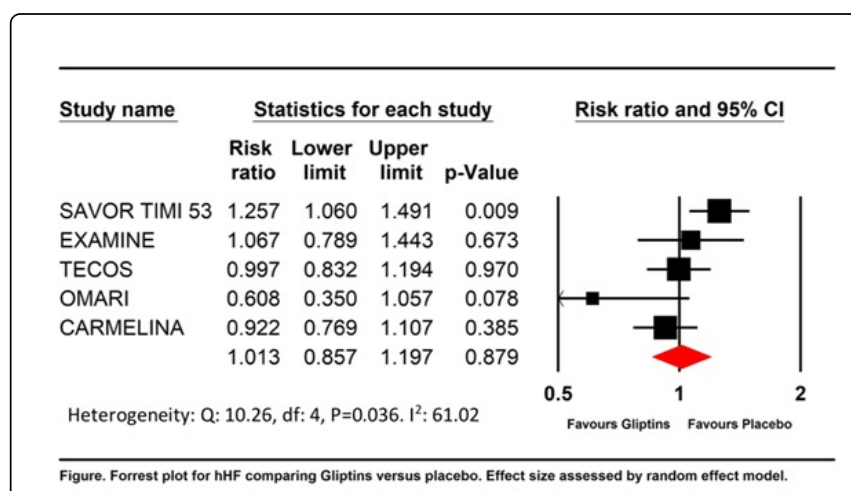

Figure 2: Forest plot for hHF comparing gliptins versus placebo. Effect size assessed by random effect model. 


\section{Discussion}

This meta-analysis reassures the medical fraternity as far as gliptins and hospitalization due to heart failure is concerned in diabetic patients with established cardiovascular disease or high CV risk.

\section{Study limitations}

We need to appreciate the limitations associated with including both SAVOR-TIMI 53 (large effect size skewing the data) and truncated omarigliptin data (very small number of events). This resulted in increased heterogeneity as expected. To overcome the impact on the analysis we selected the random effect model. Another limitation of this meta-analysis has been analyzing aggregate data instead of patient-related data.

This limits our ability to dig deeper into subgroups and analyze them separately. However, the inclusion of a common end-point definition helped us overcoming a lot of these limitations.

The question remains whether gliptins can be safely prescribed in patients with type 2 diabetes and high CV risk. Recent analysis of the saxagliptin data from SAVOR-TIMI 53 by Sheen AJ counters the data which emerged from the original study [16]. A very similar analysis of $\mathrm{hHF}$ and alogiptin questions the findings from the EXAMINE trial [17].

However, another recent review on gliptins and hHF by Packer came up with a diametrically opposite conclusion practically endorsing the universal labelling [18]. A pharmacovigilance data also seems to suggest increased hHF risk with an additional caution regarding a bias related to increased reporting noticed after the publication of SAVORTIMI 53 trial [19].

\section{Future Directions}

With the conflicting evidence at hand there can be a few ways to put this issue at rest. One option is to conduct a dedicated outcomes trial with hHF as the primary end point. Additional options include, a meta-analysis with all the relevant CVOTs as in this case or a mechanistic study exploring the pathophysiological basis for gliptins causing heart failure hospitalizations.

Mechanistic Evaluation of Glucose-lowering Strategies in Patients with Heart Failure (MEASURE-HF) is a 24 week, double-blind, randomized, multi-centric placebo-controlled study $(\mathrm{N}=330)$ is currently undergoing which is investigating the effects of saxagliptin and sitagliptin on cardiac dimensions and function (change in LVEDV index measured by MRI) in patients with type 2 diabetes and heart failure. This study might enlighten us about differential hHF with gliptins once it is completed in 2019 [20].

\section{Conclusion}

At present these are the only CVOTs we have and we need to do the best with the available data. The more CV outcomes data we seem to add on to SAVOR-TIMI 53 for analysis of hHF, the more the statistical significance seems to disappear. This meta-analysis does not point at a statistical significant increased risk of hHF in patients with type 2 diabetes and high CV risk.

Our data with the recent reviews on the same topic seems to indicate a safety profile for this group of drugs as far as the hHF signal is concerned. However more definitive data (both mechanistic \& clinical) needs to be accumulated in the future.

\section{Declaration of Interests}

There are no conflicts of interest declared.

\section{References}

1. Lamb T (2018) DPP-4 Diabetes Drug Side Effects Include Heart Attacks And Strokes As Well As Heart Failure. Drug Injury Watch.

2. Nissen SE, Wolski K (2007) Effect of rosiglitazone on the risk of myocardial infarction and death from cardiovascular causes. $\mathrm{N}$ Engl $\mathrm{J}$ Med 356: 2457- 2471.

3. U.S. Department of Health and Human Services and Food and Drug Administration Center for Drug Evaluation and Research (CDER) (2008) Guidance for Industry Diabetes Mellitus-Evaluating Cardiovascular Risk in New Antidiabetic Therapies to Treat Type 2 Diabetes.

4. Scirica BM, Bhatt DL, Braunwald E, Steg PG, Davidson J, et al. (2013) Saxagliptin and cardiovascular outcomes in patients with type 2 diabetes mellitus. N Engl J Med 369: 1317-1326.

5. http://www.fda.gov/ohrms/dockets/ac/09/briefing/2009-4422b1-01FDA.pdf

6. White WB, Cannon CP, Heller SR, Nissen SE, Bergenstal RM, et al. (2013) Alogliptin after acute coronary syndrome in patients with type 2 diabetes. N Engl J Med 369: 1327-1335.

7. Green JB, Bethel MA, Armstrong PW, Buse JB, Engel SS, et al. (2015) Effect of sitagliptin on cardiovascular outcomes in type 2 diabetes. $\mathrm{N}$ Engl J Med 373: 232-242.

8. Kongwatcharapong J, Dilokthornsakul P, Nathisuwan S, Phrommintikul A, Chaiyakunapruk N (2016) Effect of dipeptidyl peptidase-4 inhibitors on heart failure: A meta-analysis of randomized clinical trials. Int J Cardiol 211: 88-95.

9. Abbas AS, Dehbi HM, Ray KK (2016) Cardiovascular and noncardiovascular safety of dipeptidyl peptidase-4 inhibition: a meta-analysis of randomized controlled cardiovascular outcome trials. Diabetes Obes Metab18: 295-299.

10. Li L, Li S, Deng K, Liu J, Vandvik PO, et al. (2016) Dipeptidyl peptidase-4 inhibitors and risk of heart failure in type 2 diabetes: systematic review and meta-analysis of randomized and observational studies. BMJ 352: i610.

11. Elgendy IY, Mahmoud AN, Barakat AF, Elgendy AY, Saad M, et al. (2017) Cardiovascular safety of dipeptidyl peptidase IV inhibitors: a metaanalysis of placebo-controlled randomized trials. Am J Cardiovasc Drugs 17: 143-155.

12. Verma S, Goldenberg RM, Bhatt DL, Farkouh ME, Quan A, et al. (2017) Dipeptidyl peptidase-4 inhibitors and the risk of heart failure: a systematic review and meta-analysis. CMAJ open 5: E152-E177.

13. Barry AR, Turgeon RD (2016) DPP-4 Inhibitors: The Seinfeld of Oral Antihyperglycemics. Can J Hosp Pharm 69: 253-254.

14. Gantz I, Chen M, Suryawanshi S, Ntabadde C, Shah S, et al. (2017) A randomized, placebo-controlled study of the cardiovascular safety of the once-weekly DPP-4 inhibitor omarigliptin in patients with type 2 diabetes mellitus. Cardiovasc Diabetol 16: 112.

15. Eli Lilly and Company, Boehringer Ingelheim (2018) Boehringer Ingelheim and Lilly present full results of Tradjenta ${ }^{{ }^{\prime}}$ C CARMELINA cardiovascular outcome trial.

16. Sheen AJ (2018) The safety of gliptins: updated data in 2018. Expert Opin Drug Saf 17: 387-405.

17. Sharma A, Cannon CP, White WB, Liu Y, Bakris GL, et al. (2018) Early and Chronic DipeptidylPeptidaseIV Inhibition and Cardiovascular Events in Patients with Type 2 Diabetes Mellitus After an Acute Coronary Syndrome: A Landmark Analysis of the EXAMINE Trial. J Am Heart Assoc 7: e007649. 
Citation: Ghosal S, Singh AK (2018) Hospitalisation Due to Heart Failure with Gliptins and Universal Label Change (FDA)-Still Justified? A MetaAnalysis of 5 Cardiovascular Outcomes Trials. J Diabetes Metab 9: 812. doi:10.4172/2155-6156.1000812

Page 4 of 4

18. Packer M (2018) Worsening Heart Failure During the Use of DPP-4 Inhibitors Pathophysiological Mechanisms, Clinical Risks, and Potential Influence of Concomitant Antidiabetic Medications. JACC: Heart Failure 807: 445-451.

19. Fadini GP, Sarandhar M, Avogaro A (2018) Pharmacovigilance Evaluation of the Association between DPP-4 Inhibitors and Heart
Failure: Stimulated Reporting and Moderation by Drug Interactions. Diabetes Ther 9: 851-861.

20. Mechanistic Evaluation of Glucose-lowering Strategies in Patients with Heart Failure (MEASURE-HF). 2018. 